http://dx.doi.org/10.1590/0104-1428.1598

\title{
Influência das Condições de Mistura e dos Teores de Acetato de Vinila e de Argila nas Propriedades de Nanocompósitos de EVA/Montmorilonita Organofílica
}

\author{
Daiane Dias de Queiroz, Patrícia Moraes Sinohara Souza, Júlia Eulálio de Souza Bertucci, \\ Amanda de Freitas Mendes Vieira, Ana Rita Morales \\ Departamento de Engenharia de Materiais e de Bioprocessos - DEMBio, Faculdade de Engenharia \\ Química, Universidade Estadual de Campinas \\ Claire Isabel Grígoli de Luca Sarantopoulos \\ Centro de Tecnologia de Embalagem - CETEA, Instituto de Tecnologia de Alimentos
}

\begin{abstract}
Resumo: O copolímero Etileno Acetato de Vinila (EVA) é uma poliolefina utilizada principalmente em filmes para embalagens, onde a sua flexibilidade, tenacidade, elasticidade e transparência são atributos desejáveis. As propriedades mecânicas, térmicas e de barreira do EVA podem ser melhoradas pela incorporação de cargas inorgânicas. O EVA apresenta polaridade variável de acordo com o teor de Acetato de Vinila, o que promove a viabilidade para obtenção de nanocompósitos com argilas organicamente modificadas, tornando desnecessária a adição de agentes compatibilizantes. Neste trabalho foram preparados nanocompósitos de EVA e montmorilonita organofílica comercial pelo método de intercalação do fundido em um reômetro de torque, com rotores tipo roller, a $150^{\circ} \mathrm{C}$ durante 10 minutos, com monitoramento do torque em função do tempo. A fim de investigar a influência da polaridade do EVA, da concentração da argila e das condições de processamento nas características dos nanocompósitos, variou-se a concentração de Acetato de Vinila (19\% e 28\%), o teor de argila (2\% e 5\%) e a velocidade de rotores (60 rpm e $100 \mathrm{rpm})$. Foram avaliadas a estrutura da argila, a estabilidade térmica e as propriedades mecânicas e de barreira dos nanocompósitos, mediante as técnicas de difração de raios $\mathrm{X}$, análise termogravimétrica, ensaio de tração e análises de taxa de permeabilidade ao oxigênio e ao vapor d'água. A partir dos dados de permeabilidade foi aplicado o modelo de Nielsen para avaliar o grau de esfoliação da argila. Dentre os principais resultados com o planejamento fatorial destacam-se: (a) o aumento da polaridade do EVA aumentou a permeabilidade ao oxigênio e ao vapor d'água dos materiais; (b) o teor de argila não modificou a estabilidade térmica dos nanocompósitos e a estrutura da argila; (c) o aumento da concentração da argila aumentou o módulo elástico, reduziu a deformação de ruptura e reduziu significativamente o coeficiente de permeabilidade ao oxigênio e ao vapor d'água; (d) o fator rotação não implicou em alterações significativas em nenhuma das respostas avaliadas.
\end{abstract}

Palavras-chave: Nanocompósitos, EVA, permeabilidade, montmorilonita organofilica.

\section{Influence of Processing and Content of Vinyl Acetate on the Properties of Eva/ Organophilic Montmorillonite Nanocomposites}

\begin{abstract}
The copolymer Ethylene Vinyl Acetate (EVA) is a polyolefin used mainly in packaging films, where its flexibility, toughness, elasticity and transparency are desirable attributes. The mechanical, thermal and barrier properties of EVA can be improved by the incorporation of inorganic fillers. Its polarity varies according to the content of Vinyl Acetate, which allows for nanocomposites to be obtained with organically modified clay, with no need to add compatibilizing agents. In this work nanocomposites of EVA and commercial organophilic montmorillonite were prepared by melt intercalation in a torque rheometer with rotors roller type, at $150^{\circ} \mathrm{C}$ for 10 minutes, monitoring the torque versus time. The concentration of Vinyl Acetate (19\% and 28\%), clay content (2\% to 5\%) and the rotors rotation (60 rpm to 100 $\mathrm{rpm}$ ) were varied in order to investigate their influence on the characteristics of the nanocomposites. The clay structure, thermal stability, mechanical and barrier properties of the nanocomposites were evaluated by X-ray diffraction, thermal analysis, tensile testing, oxygen and water vapor transmission rates. Based on the permeability data, Nielsen's model was applied to assess the degree of clay exfoliation. The main results obtained from the factorial design are: (a) increasing the polarity of EVA led to an increase in the permeability to oxygen and water vapor of the material, (b) the clay content did not change the clay structure or the thermal stability of the nanocomposites, (c) increasing the concentration of clay increased the elastic modulus, decreased the elongation at break and significantly reduced the coefficient of permeability for oxygen and water vapor, (d) the rotation factor did not result in significant changes in any of the evaluated responses.
\end{abstract}

Keywords: Nanocomposites, EVA, permeability, organophilic montmorillonite.

Autor para correspondência: Ana Rita Morales, Departamento de Engenharia de Materiais e de Bioprocessos - DEMBio, Faculdade de Engenharia Química, Universidade Estadual de Campinas - UNICAMP, Av. Albert Einstein, 500, Cidade Universitária Zeferino Vaz, CEP 13083-852, Campinas, SP, Brasil, e-mail: morales@feq.unicamp.br 


\section{Introdução}

Nos últimos anos o campo de aplicação dos polímeros tem sido largamente ampliado, ocupando espaços antes pertencentes a outros materiais como as cerâmicas e os metais, despertando grande interesse em pesquisas tecnológicas e acadêmicas. Estas novas aplicações necessitam de propriedades que muitas vezes o polímero puro não apresenta e são obtidas por meio de modificações, que podem ser realizadas com a incorporação de nanopartículas de cargas inorgânicas como argila, carbonato de cálcio, nanotubos de carbono entre outros, dando origem a uma nova classe de materiais poliméricos, os nanocompósitos ${ }^{[1]}$.

A fase inorgânica mais utilizada na preparação de nanocompósitos poliméricos é a argila montmorilonita de origem natural, cuja razão de aspecto é bastante elevada. A montmorilonita apresenta boa capacidade de delaminação somada à alta resistência a solventes e estabilidade térmica necessária aos processos de polimerização e de extrusão, fatores que levaram à sua popularização como carga para nanocompósitos com eficácia comprovada ${ }^{[2]}$.

As argilas tendem a ser hidrofílicas e os polímeros são organofílicos, o que impede uma boa dispersão da argila na matriz polimérica. No entanto, a compatibilização entre as superfícies da argila e do polímero pode ser feita através da modificação superficial da argila com surfactantes catiônicos, na qual ocorre a substituição dos cátions inorgânicos por cátions orgânicos de cadeias longas $^{[3]}$. Como resultado da modificação orgânica, as argilas são intercaladas com os cátions orgânicos, o que aumenta o espaçamento entre as camadas e melhora a compatibilidade da argila com a matriz polimérica ${ }^{[4]}$. Entre as técnicas de preparação de nanocompósitos de polímero e argilas organicamente modificadas, destaca-se a técnica de intercalação do fundido, na qual os componentes são misturados com o polímero no estado fundido. Este método é adequado para uso industrial e ambientalmente correto por não requerer a utilização de solventes ${ }^{[4,5]}$.

O copolímero etileno Acetato de Vinila (EVA) pertence ao grupo das poliolefinas e é muito utilizado na fabricação de embalagens para carne, liners para tampas de bebidas carbonatadas, na construção civil e na fabricação de fios e cabos. Sua polaridade varia de acordo com o teor de Acetato de Vinila ${ }^{[6]}$. Devido à polaridade do copolímero etileno Acetato de Vinila (EVA) pode-se obter uma boa dispersão da argila, sendo desnecessária a adição de agentes compatibilizantes, resultando em melhorias nas propriedades de barreira e propriedades mecânicas. Trabalhos com nanocompósitos de argila e EVA relatam que a morfologia mais observada para estes sistemas é uma mistura de estruturas esfoliadas e intercaladas ${ }^{[7-9]}$, sendo que suas propriedades dependem, principalmente, desta morfologia resultante. Relata-se que a formação de morfologia intercalada e/ou esfoliada está relacionada com o tipo e quantidade de mineral de argila, o teor de Acetato de Vinila (VA) na matriz de EVA e as condições de processamento ${ }^{[10]}$

O modelo de Nielsen tem sido aplicado para descrever de forma simples o nível de dispersão de nanoplaquetas em uma membrama através das medidas de permeabilidade relativa entre um sistema com nanoplaquetas uniformemente dispersas e um mesmo sistema puro ${ }^{[11]}$. O modelo assume que as nanoplaquetas agem como barreiras impermeáveis, criando um caminho tortuoso para a difusão das moléculas e correlaciona o coeficiente de permeabilidade $(\mathrm{P})$ com a razão de aspecto $(\alpha)$ das nanoplaquetas segundo a Equação $1^{[12,13]}$.

$$
\frac{\mathrm{P}_{\text {nanocompósito }}}{\mathrm{P}_{\text {matriz }}}=\frac{1-\phi}{1+\frac{\alpha \phi}{2}}
$$

Onde: $\phi$ é a fração volumétrica das nanoplaquetas e $\alpha=\mathrm{L} / \mathrm{W}, \mathrm{L}=$ comprimento e $\mathrm{W}=$ espessura das nanoplaquetas;

Outros fatores, como orientação das camadas impermeáveis do sistema e forma geométrica das partículas, têm também influência na permeabilidade e modelos foram propostos considerando estas variáveis, no entanto, o modelo simplificado de Nielsen é bastante aceito para a previsão da permeabilidade de nanocompósitos de $\operatorname{argilas}^{[14]}$. O processamento utilizado para o sistema em estudo não tem a característica de conferir alto grau de orientação, e sendo a mesma argila utilizada para todas as amostras, considerou-se adequado o Modelo de Nielsen.

Embora existam vários trabalhos sobre nanocompósitos poliméricos com argilas organofílicas, poucos utilizaram o reômetro de torque para acompanhar o processo de incorporação das argilas em EVA puro. Este estudo visa estudar a influência dos teores de Acetato de Vinila no EVA e argila e das condições de mistura na estrutura da argila e nas propriedades mecânicas, térmicas e na permeabilidade ao oxigênio e ao vapor d'água dos nanocompósitos.

\section{Experimental}

Para a preparação dos nanocompósitos foram utilizados dois tipos de copolímero Etileno Acetato de Vinila (EVA): um contendo $28 \%$ de Acetato de Vinila (VA), índice de fluidez (MFI) $6 \mathrm{~g} / 10 \mathrm{~min}$ e massa específica $0,95 \mathrm{~g} / \mathrm{cm}^{3}$ (HM728, Braskem) (EVA 28) e o outro com 19\% de (VA), MFI de $8 \mathrm{~g} / 10$ min e massa específica $0,94 \mathrm{~g} / \mathrm{cm}^{3}$ (8019PE, Braskem) (EVA 19). Foram escolhidos estes materiais com MFI próximos para minimizar o efeito da massa molar e direcionar a análise para o teor de VA da resina de EVA. Também foi utilizado um masterbatch de argila organofílica/EVA que contém $25 \%$ de argila organofílica e $75 \%$ de EVA, com $28 \%$ de VA, apresentando MFI de 35-38 g/10min, (NANOMAX®-EVA, Nanocor). Segundo informações do fabricante, o masterbatch é produzido com a argila nanomer 1.44P, também da Nanocor. O NANOMAX EVA é um material comercial, no qual a argila já se encontra pré dispersa, o que melhora as condições de homogeneização desta na resina. Embora o veículo do masterbatch apresente maior teor de VA e menor MFI, sua concentração final limita-se a aproximadamente 3,8\% no produto final e seus efeitos nos resultados tendem a ser o mesmo para as duas diferentes resinas. Os nanocompósitos foram obtidos pelo método de intercalação do fundido. O masterbatch foi diluído nas resinas estudadas nas concentrações desejadas em um reômetro de torque da marca Brabender (model mixer 30/50E, Germany), com 
rotores tipo roller, operando a $150^{\circ} \mathrm{C}$, durante $10 \mathrm{~min}$. Em seguida foram obtidas amostras com espessura em torno de $0,3 \mathrm{~mm}$ em uma prensa $\mathrm{MH}$, modelo Q/FMot8ton, com moldagem a $210^{\circ} \mathrm{C}$ seguido por prensagem a frio por resfriamento com circulação de água. Para o ensaio de tração foram cortados corpos de prova por estampagem segundo a ASTM 638-10.

A influência da polaridade do EVA (teor de VA), do teor de argila e das condições de processamento nas características dos nanocompósitos foi estudada mediante um planejamento experimental $2^{3}$. Foram preparadas 8 formulações, a fim de se avaliar a influência de 3 variáveis em 2 níveis. As variáveis avaliadas foram: $\mathrm{X}_{1}=$ teor de argila (nível superior: $5 \%$; nível inferior: $2 \%$ ); $\mathrm{X}_{2}=$ rotação (nível superior: $100 \mathrm{rpm}$; nível inferior: $60 \mathrm{rpm}$ ) e $\mathrm{X}_{3}=$ concentração de Acetato de Vinila (nível superior: 28\%; nível inferior: 19\%). As amostras foram identificadas em função das variáveis como $\mathrm{X}_{1} / \mathrm{X}_{2} / \mathrm{X}_{3}$.

Para os filmes de nanocompósitos foi realizada análise estrutural da argila mediante a técnica de difração de raios- X. A estabilidade térmica, as propriedades mecânicas e permeabilidade foram avaliadas por análise termogravimétrica, ensaios de tração e de taxas de permeabilidade ao oxigênio e ao vapor d'água, respectivamente.

A análise de dispersão da argila nos nanocompósitos foi realizada pela técnica de difração de raios-X (DRX), utilizando-se um difratômetro de raios-X Philips (modelo PW1710, Holanda) com varredura de $1,75^{\circ}$ a $10^{\circ}(2 \theta)$, radiação de $\mathrm{K} \alpha$ do $\mathrm{Cu}\left(\lambda=1,54 \mathrm{~A}^{\circ}\right)$, tensão de $40 \mathrm{kV}$ e corrente $30 \mathrm{~mA}$. Para avaliar a estabilidade térmica das amostras foi realizada a análise termogravimétrica (TGA), em um equipamento TA Instruments modelo SDT 2960 a uma taxa de aquecimento de $10^{\circ} \mathrm{C} / \mathrm{min}$, a partir da temperatura ambiente até $550^{\circ} \mathrm{C}$, sob atmosfera de gás nitrogênio.

Os ensaios de tração foram realizados de acordo com a norma ASTM D638-10, em uma Máquina Universal de Ensaios, marca MTS, modelo Aliance RT/5, com célula de carga de $100 \mathrm{~N}$ e velocidade de $100 \mathrm{~mm} / \mathrm{min}$. A taxa de permeabilidade ao oxigênio $\left(\mathrm{TPO}_{2}\right)$ dos filmes foi determinada por método coulométrico, segundo a norma ASTM D3985(ASTM INTERNATIONAL, 2010), em equipamento OXTRAN, modelo $2 / 20$, da MOCON, operando com oxigênio puro como gás permeante, a $23^{\circ} \mathrm{Ce}$ a seco. A área efetiva de permeação de cada corpo de prova foi de $100 \mathrm{~cm}^{2}$. Os resultados obtidos foram corrigidos para latm de gradiente de pressão parcial de oxigênio, entre as duas superfícies do filme.

A taxa de permeabilidade ao vapor d'água (TPVA) dos filmes foi avaliada por método gravimétrico, segundo a norma ASTM E96 /E96M (ASTM INTERNATIONAL, 2012), em cápsulas de alumínio vedadas com uma mistura $60 \%$ de cera microcristalina com $40 \%$ de parafina cristalina refinada. Cloreto de Cálcio Anidro $\left(\mathrm{CaCl}_{2}\right)$ foi utilizado como dessecante. As cápsulas foram colocadas dentro de uma estufa - marca 'Omron' e modelo 'E5CX' a $38^{\circ} \mathrm{C}$ e umidade relativa de $90 \%$. A umidade relativa foi mantida por meio da solução salina saturada de cloreto de bário $\left(\mathrm{BaCl}_{2}\right.$.2H20). Uma vez determinadas a $\mathrm{TPO}_{2}$ e a TPVA, calculou-se o coeficiente de permeabilidade $(\mathrm{P})$ a partir da taxa de permeabilidade, segundo a Equação 2.

$$
\mathrm{P}=\text { TP.e } / \mathrm{p}
$$

Onde: $\mathrm{P}=$ coeficiente de permeabilidade; $\mathrm{TP}=$ taxa de permeabilidade ao oxigênio ou ao vapor d'água; $\mathrm{e}=$ espessura média do corpo-de-prova e $\mathrm{p}=$ gradiente de pressão do permeante entre as superfícies do filme.

Os cálculos realizados para determinação da influência dos fatores sobre as propriedades do material foram realizados utilizando o software Statistica 7.0, a 95\% de nível de confiança.

\section{Resultados e Discussão}

\section{Processo de incorporação de argila}

A partir das curvas torque $\mathrm{x}$ tempo obtidas durante o processamento dos nanocompósitos de EVA/argila organofílica, foram determinados os valores médios dos parâmetros: torque no pico de carregamento $(\mathrm{Tc})$, torque mínimo $(\mathrm{Tm})$ e tempo de fusão $\left(\mathrm{t}_{\mathrm{f}}\right)$, para cada formulação (Tabela 1). Nesta mesma tabela estão os valores de DRX, discutidos em sequência.

A reometria de torque foi fundamental para a obtenção e amostras uniformes. Os valores de torque no pico de carregamento e do tempo de fusão apresentaram pequena variação entre as diferentes formulações estudadas, indicando pouca variação nas condições de carregamento dos materiais na câmera de mistura. A medição de Tm é importante pois indica de maneira indireta a viscosidade do material após atingir o estado estacionário. A variável de estudo, rotação, não causou alteração significativa de Tm. As amostras com $28 \%$ de VA fundiram mais rapidamente que as amostras com 19\% de VA. O teor de argila poderia aumentar os valores de Tm, pelo aumento de viscosidade esperado, mas isto não foi observado para as concentrações aqui estudadas.

\section{Análise estrutural}

Os difratogramas comparativos entre as amostras de nanocompósitos e o masterbatch estão representados na Figura 1A (formulações com EVA 19\%) e na Figura 1B (formulações com EVA 28\%).

Tabela 1. Resultados dos parâmetros da curva de torque $\mathrm{x}$ tempo e espaçamento basal $\mathrm{d}_{001}$.

\begin{tabular}{lcccc}
\hline \multicolumn{1}{c}{ Amostra $^{*}$} & Tc $(\mathbf{N} . m)$ & Tm $(\mathbf{N} . m)$ & tf $(\mathbf{m i n})$ & $\mathbf{d}_{\mathbf{0 0 1}}(\mathbf{n m})$ \\
\hline $\operatorname{EVA}(2 / 60 / 19)$ & $20,4 \pm 0,4$ & $10,3 \pm 0,4$ & $4,1 \pm 1,1$ & 3,94 \\
$\operatorname{EVA}(5 / 60 / 19)$ & $22,0 \pm 2,7$ & $10,0 \pm 0,9$ & $4,9 \pm 0,1$ & 3,76 \\
$\operatorname{EVA}(2 / 100 / 19)$ & $24,0 \pm 0,2$ & $10,5 \pm 0,2$ & $4,3 \pm 0,4$ & 3,92 \\
$\operatorname{EVA}(5 / 100 / 19)$ & $23,0 \pm 2,2$ & $10,2 \pm 0,1$ & $4,6 \pm 0,0$ & 4,30 \\
$\operatorname{EVA}(2 / 60 / 28)$ & $17,6 \pm 0,2$ & $11,4 \pm 0,3$ & $3,5 \pm 0,2$ & - \\
$\operatorname{EVA}(5 / 60 / 28)$ & $16,6 \pm 0,3$ & $10,7 \pm 0,4$ & $3,0 \pm 0,3$ & 3,88 \\
$\operatorname{EVA}(2 / 100 / 28)$ & $22,7 \pm 1,6$ & $11,1 \pm 0,1$ & $3,9 \pm 0,9$ & 3,86 \\
$\operatorname{EVA}(5 / 100 / 28)$ & $24,6 \pm 0,8$ & $11,7 \pm 0,8$ & $3,7 \pm 0,2$ & 4,08 \\
\hline
\end{tabular}

*(teor de argila/ rotação/ concentração de Acetato de Vinila); $\mathrm{Tc}=$ torque no pico de carregamento; $\mathrm{Tm}=$ torque mínimo; $\mathrm{tf}=$ tempo de fusão; $d_{001}=$ espaçamento basal. 


\section{1- $\operatorname{EVA}(2 / 60 / 19) \quad 2-\operatorname{EVA}(5 / 60 / 19)$ \\ $3-\operatorname{EVA}(2 / 100 / 19) \quad 4-\operatorname{EVA}(5 / 100 / 19)$ \\ 5- Masterbatch}

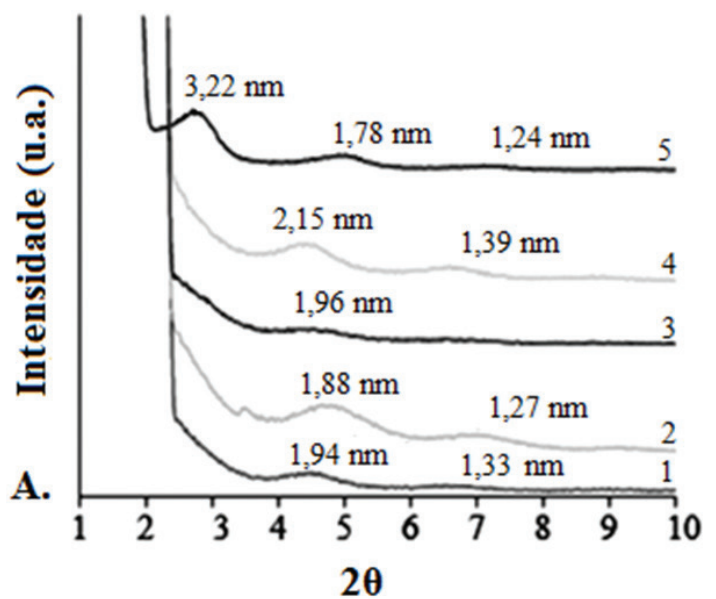

1- $\operatorname{EVA}(2 / 60 / 28) \quad 2-\operatorname{EVA}(5 / 60 / 28)$

$3-\operatorname{EVA}(2 / 100 / 28) \quad 4-\operatorname{EVA}(5 / 100 / 28)$

\section{5- Masterbatch}

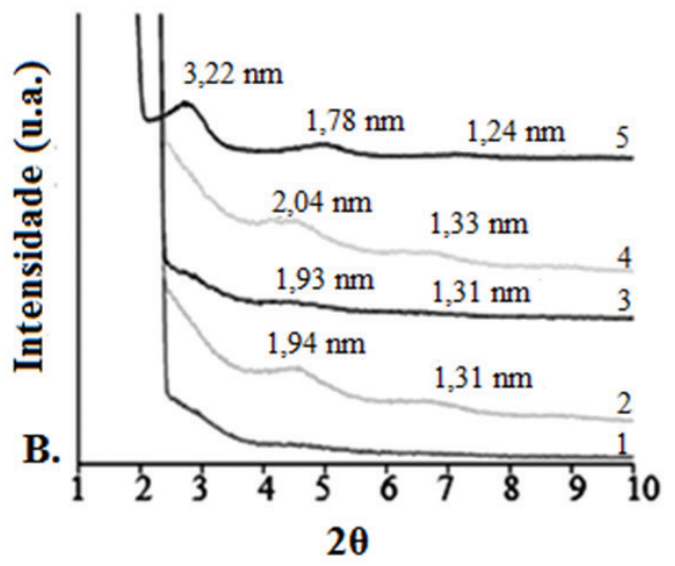

Figura 1. Difratogramas do masterbatch e dos nanocompósitos. A. EVA19: 19\% de Acetato de Vinila; B. EVA28: 28\% o de Acetato de Vinila (EVA (teor de argila/ rotação/ concentração de Acetato de Vinila)).

O padrão de difração do masterbatch indicou a presença de três picos. O primeiro, mais intenso, relacionado ao plano $\mathrm{d}_{001}$ da argila, com espaçamento basal de $3,22 \mathrm{~nm}\left(2 \theta=2,74^{\circ}\right)$ (Tabela 1). Segundo Morales et al. ${ }^{[1]}$ a argila pura Nanomer 1.44P apresenta um espaçamento basal, referente ao plano $\mathrm{d}_{001}$, de $2,28 \mathrm{~nm}\left(2 \theta=3,88^{\circ}\right)$. Os resultados indicaram que ocorreu intercalação da argila no masterbatch, o que refletiu no aumento do espaçamento basal comparado à argila pura. Os picos em torno de $2 \theta=4,96^{\circ}$ e $2 \theta=7,14^{\circ}$, também identificados no difratograma do masterbatch, correspondem aos espaçamentos de 1,78 nm e 1,24 nm, respectivamente. No caso de argilominerais é comum encontrar referências à reflexão dos planos (002) e (003) como sendo de segunda e terceira ordens do plano (001) ${ }^{[15]}$. Mandalia e Bergaya (2006) ${ }^{[16]}$ realizaram a análise de difração de raios-X para nanocompósitos de EVA e argila organofílica e consideraram os picos que ocorreram em ângulos mais altos como sendo de segunda e terceira ordem, e indicaram os valores múltiplos de espaçamento.

Com relação às formulações de nanocompósitos em estudo, notou-se a ausência de pico de primeira ordem todas as formulações. Pela análise do padrão de difração da maioria das formulações, houve o deslocamento dos picos de segunda e terceira ordem para ângulos inferiores, indicando uma estrutura intercalada de nanocompósitos. A formulação $\operatorname{EVA}(2 / 60 / 28)$, por sua vez, apresentou desaparecimento de ambos os picos, o que pode ser um indicativo de esfoliação. Shafiee e Ramazani ${ }^{[17]}$ prepararam nanocompósitos de EVA e argilas organicamente modificadas Cloisite 15A e notaram o deslocamento do pico de referência $\left(\mathrm{d}_{001} \mathrm{da}\right.$ argila) para ângulos entre $1^{\circ}$ e $2^{\circ}$. Os autores observaram que o maior teor de acetato apresentou a tendência de aumentar os valores de espaçamento basal.
Neste trabalho, o deslocamento do primeiro pico do masterbatch nos nanocompósitos também era esperado, mas sua detecção não foi possível devido ao intervalo de varredura utilizado na análise de DRX, com início em $2 \theta=1,75^{\circ}$, que é o limite do equipamento utilizado. Desta forma, o valor para o espaçamento basal, referente ao plano $\mathrm{d}_{001}$, foi estimado considerando-se o dobro do valor encontrado para o espaçamento referente ao pico de segunda ordem (Tabela 1).

Os valores de $\mathrm{d}_{001}$ indicam uma pequena variação dos valores estimados entre as formulações de estudo, com o valor mínimo obtido para formulação $\operatorname{EVA}(5 / 60 / 19)(3,76$ $\mathrm{nm})$ e valor máximo obtido para formulação $\operatorname{EVA}(5 / 100 / 28)$ $(4,3 \mathrm{~nm})$. O teor de argila não influenciou significativamente os valores o espaçamento basal. O maior teor de Acetato de Vinila poderia facilitar a intercalação da resina no espaço interlamelar da argila, propiciando estruturas mais intercaladas e/ou esfoliadas, quando comparadas às das formulações com menor teor de acetato, devido ao aumento de grupos ésteres polares na cadeia, porém, essa influência sobre o espaçamento basal não foi verificada pela análise de DRX. Marini et al. ${ }^{[18]}$ observaram que em nanocompósitos de EVA, o principal fator para obtenção de uma boa dispersão de argila não é a polaridade do polímero, mas sim sua viscosidade, sendo que quanto menor a viscosidade, maior a tendência de obter esfoliação da argila. No presente estudo, essa hipótese é coerente, levando-se em consideração a não observância da influência da polaridade sobre o espaçamento basal, e concomitantemente o fato de que os valores de MFI dos tipos de EVA utilizados são próximos, não implicando em variação significativa na viscosidade, propriedade que poderia influenciar a difusão do polímero entre as galerias de argila durante o 
processamento dos materiais. A variação da rotação aplicada na preparação das amostras também não demonstrou influência nos valores de espaçamento basal das amostras. Vale ressaltar que a técnica de DRX não é conclusiva por limitar-se à detecção das regiões onde existe ordem estrutural em concentrações detectáveis pelo equipamento, e não quantifica a distribuição de quanto da argila pode estar esfoliada ou intercalada. Por isto o interesse na aplicação do Modelo de Nielsen discutido neste trabalho.

\section{Estabilidade térmica}

Os fatores de formulação e processamento estudados (teor de argila, concentração de Acetato de Vinila e rotação) não apresentaram influência estatisticamente significativa sobre a estabilidade térmica dos nanocompósitos (parâmetros $\mathrm{T}_{5 \%} \mathrm{e} \mathrm{T}_{\text {máx }}$ apresentados na Tabela 2).

Os resultados de TGA para as amostras de EVA19 e EVA28 indicam uma primeira redução de massa em $339^{\circ} \mathrm{C}$ e $334^{\circ} \mathrm{C}$, que corresponde à pirólise dos grupos de éster acético do Acetato de Vinila com eliminação do ácido acético $^{[19]}$. Esta região de perda de massa foi acelerada para os nanocompósitos, como pode ser observado pela redução da temperatura de perda de $5 \%$ em massa, o que pode ser atribuído à degradação do modificador orgânico ${ }^{[20]}$. Notouse ainda uma segunda região de perda de massa (acima de

Tabela 2. Temperatura de decomposição para o EVA puro e nanocompósitos.

\begin{tabular}{lcc}
\hline \multicolumn{1}{c}{ Amostra } & $\mathbf{T}_{\mathbf{5 \%}}\left({ }^{\circ} \mathbf{C}\right)$ & $\mathbf{T}_{\text {máx }}\left({ }^{\circ} \mathbf{C}\right)$ \\
\hline $\operatorname{EVA} 19$ & 339 & 470 \\
$\operatorname{EVA}(2 / 60 / 19)$ & 327 & 463 \\
$\operatorname{EVA}(5 / 60 / 19)$ & 326 & 454 \\
$\operatorname{EVA}(2 / 100 / 19)$ & 329 & 459 \\
$\operatorname{EVA}(5 / 100 / 19)$ & 321 & 454 \\
$\operatorname{EVA28}$ & 334 & 467 \\
$\operatorname{EVA}(2 / 60 / 28)$ & 324 & 468 \\
$\operatorname{EVA}(5 / 60 / 28)$ & 319 & 455 \\
$\operatorname{EVA}(2 / 100 / 28)$ & 327 & 470 \\
$\operatorname{EVA}(5 / 100 / 28)$ & 323 & 456 \\
\hline
\end{tabular}

$\mathrm{T}_{5 \%} \%$ : temperatura de perda de $5 \%$ em massa; $\mathrm{T}_{\text {máx }}$ : temperatura de degradação do polímero; EVA19: 19\% de Acetato de Vinila; EVA28: $28 \%$ o de Acetato de Vinila; EVA (teor de argila/ rotação/ concentração de Acetato de Vinila. $400^{\circ} \mathrm{C}$ ), que se refere à degradação do copolímero resultante da desacetilação ${ }^{[19]}$.

\section{Propriedades de tração}

Os resultados dos ensaios de tração para as amostras de EVA puro e os nanocompósitos com montmorilonita organofílica estão resumidos na Tabela 3 .

Os fatores avaliados pelo planejamento fatorial (teor de argila, rotação e concentração de Acetato de Vinila) apresentaram influência estatisticamente significativa sobre as propriedades mecânicas. Os diagramas de Pareto são apresentados na Figura 2.

Observou-se que o aumento no teor de Acetato de Vinila provocou uma diminuição no módulo elástico e aumento na deformação de ruptura, o que já era esperado, visto que o aumento do teor de Acetato de Vinila implica em redução da cristalinidade, e consequentemente, diminuição da rigide ${ }^{[21]}$. O teor de argila teve um comportamento totalmente oposto com significância positiva no aumento do módulo elástico e redução na deformação de ruptura. Chaudhary et al. ${ }^{[22]}$ observaram um comportamento semelhante em seu estudo. Os autores notaram que com o aumento do teor de argila em nanocompósitos com EVA 18\%, o módulo aumentava significativamente. Já para os nanocompósitos com EVA 28\%, apesar destes apresentarem morfologia similar aos nanocompósitos com EVA $18 \%$, os valores de módulo apresentaram um aumento muito pequeno, e apontaram que o grau de amorfismo do polímero apresenta uma influência sobre a rigidez dos nanocompósitos ainda maior do que a própria interação polímero-argila. Considerando-se os valores da Tabela 3, as propriedades de resistência à tração e deformação na ruptura, por sua vez, foram pouco influenciadas pela incorporação de reforço na matriz de EVA, o que está coerente com os relatos de La Mantia et al. ${ }^{[23]}$ que também observaram este efeito de aumentar a rigidez do material sem implicar em perdas em outras propriedades diferindo de forma vantajosa em relação aos microcompósitos convencionais, que em geral aumentam a rigidez do material, mas com um acentuado decréscimo da deformação na ruptura.

Com relação ao parâmetro rotação, os diagramas de Pareto revelam que houve influência negativa sobre a resistência à tração, o que pode estar associado ao processo de degradação da amostra. A deformação na ruptura, por sua vez, foi influenciada de forma positiva.

Tabela 3. Resultados dos ensaios de tração para o EVA puro e nanocompósitos.

\begin{tabular}{|c|c|c|c|}
\hline Amostra & Módulo de Elasticidade (MPa) & Resistência à Tração (MPa) & Deformação na Ruptura (\%) \\
\hline EVA19 & $12 \pm 1$ & $16 \pm 0$ & $731 \pm 22$ \\
\hline $\operatorname{EVA}(2 / 60 / 19)$ & $29 \pm 4$ & $14 \pm 1$ & $739 \pm 26$ \\
\hline $\operatorname{EVA}(5 / 60 / 19)$ & $34 \pm 5$ & $13 \pm 1$ & $717 \pm 33$ \\
\hline $\operatorname{EVA}(2 / 100 / 19)$ & $19 \pm 1$ & $13 \pm 1$ & $778 \pm 29$ \\
\hline $\operatorname{EVA}(5 / 100 / 19)$ & $57 \pm 6$ & $12 \pm 1$ & $696 \pm 30$ \\
\hline EVA28 & $3 \pm 0$ & $16 \pm 1$ & $878 \pm 48$ \\
\hline $\operatorname{EVA}(2 / 60 / 28)$ & $3 \pm 0$ & $15 \pm 1$ & $910 \pm 30$ \\
\hline $\operatorname{EVA}(5 / 60 / 28)$ & $5 \pm 0$ & $18 \pm 1$ & $704 \pm 26$ \\
\hline $\operatorname{EVA}(2 / 100 / 28)$ & $3 \pm 0$ & $16 \pm 1$ & $921 \pm 50$ \\
\hline $\operatorname{EVA}(5 / 100 / 28)$ & $3 \pm 0$ & $14 \pm 0$ & $904 \pm 28$ \\
\hline
\end{tabular}

EVA19: 19\% de Acetato de Vinila; EVA28: 28\% o de Acetato de Vinila; EVA (teor de argila/ rotação/ concentração de Acetato de Vinila. 


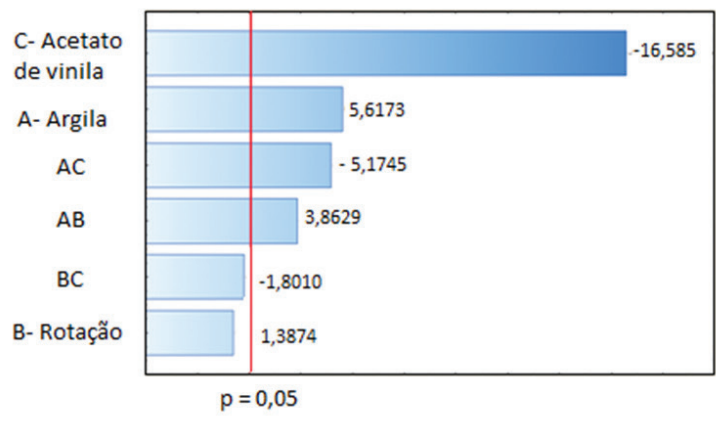

(a)

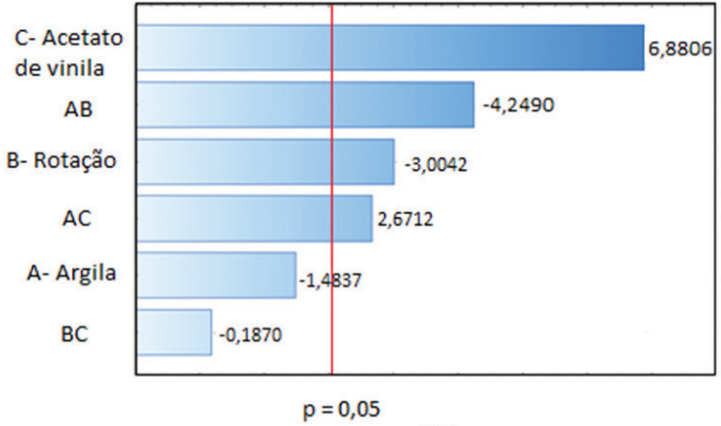

(b)

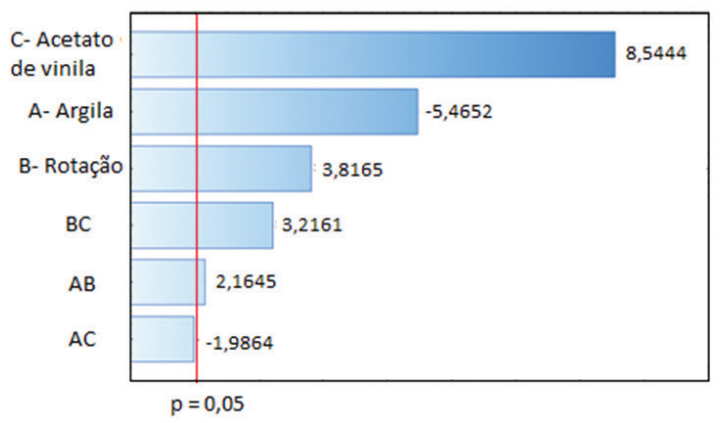

(c)

Figura 2. Diagramas de Pareto para as propriedades mecânicas. (a) Módulo elástico; (b) Resistência à tração; (c) Deformação na ruptura.

Tabela 4. Valores de taxa de permeabilidade ao oxigênio $\left(\mathrm{TPO}_{2}\right)$ e vapor d'água(TPVA) do EVA19, EVA28 e seus nanocompósitos.

\begin{tabular}{|c|c|c|c|c|c|c|}
\hline Amostras & $\begin{array}{c}\mathrm{TPO}_{2}(\mathrm{~mL} \text { (CNTP). } \\
\left.\mathrm{m}^{-2} \cdot \mathrm{dia}^{-1}\right)\end{array}$ & TPVA (g. m $\left.{ }^{-2} \cdot \operatorname{dia}^{-1}\right)$ & $\begin{array}{c}\mathrm{PO}_{2}(\mathrm{~mL}(\mathrm{CNTP}) . \\
\left.\mu \mathrm{m} . \mathrm{m}^{-2} \cdot \mathrm{dia}^{-1} \cdot \mathrm{atm}^{-1}\right)\end{array}$ & $\begin{array}{c}\text { PVA (g. mm. min }{ }^{-2} . \\
\left.\text { dia }^{-1} \cdot a^{-1 m^{-1}}\right)\end{array}$ & $\begin{array}{l}\text { Redução } \\
\mathrm{PO}_{2}(\%)\end{array}$ & $\begin{array}{l}\text { Redução } \\
\text { PVA (\%) }\end{array}$ \\
\hline EVA19 & $1294 \pm 191$ & $18 \pm 4$ & $389 \pm 10$ & $5,1 \pm 0,4$ & - & - \\
\hline $\operatorname{EVA}(2 / 60 / 19)$ & $1024 \pm 124$ & $13 \pm 6$ & $285 \pm 45$ & $3,0 \pm 1,2$ & 7 & 28 \\
\hline $\operatorname{EVA}(5 / 60 / 19)$ & $824 \pm 7$ & $12 \pm 2$ & $209 \pm 6$ & $3,2 \pm 2,1$ & 36 & 33 \\
\hline $\operatorname{EVA}(2 / 100 / 19)$ & $1115 \pm 0$ & $8 \pm 0$ & $320 \pm 0$ & $2,8 \pm 0,1$ & 14 & 55 \\
\hline $\operatorname{EVA}(5 / 100 / 19)$ & $914 \pm 162$ & $7 \pm 1$ & $199 \pm 23$ & $1,9 \pm 0,1$ & 29 & 61 \\
\hline EVA28 & $1575 \pm 124$ & $24 \pm 2$ & $454 \pm 33$ & $8,4 \pm 0,4$ & - & - \\
\hline $\operatorname{EVA}(2 / 60 / 28)$ & $960 \pm 198$ & $14 \pm 6$ & $413 \pm 43$ & $5,6 \pm 2,0$ & 39 & 42 \\
\hline $\operatorname{EVA}(5 / 60 / 28)$ & $497 \pm 91$ & $10 \pm 3$ & $244 \pm 16$ & $3,1 \pm 0,4$ & 68 & 58 \\
\hline $\operatorname{EVA}(2 / 100 / 28)$ & $1111 \pm 13$ & $13 \pm 1$ & $376 \pm 5$ & $4,6 \pm 0,7$ & 29 & 46 \\
\hline $\operatorname{EVA}(5 / 100 / 28)$ & $580 \pm 46$ & $11 \pm 1$ & $249 \pm 13$ & $4,0 \pm 0,4$ & 63 & 54 \\
\hline
\end{tabular}

EVA19: 19\% de Acetato de Vinila; EVA28: 28\% o de Acetato de Vinila; EVA (teor de argila/ rotação/ concentração de Acetato de Vinila. Embora a taxa de permeabilidade ao oxigênio seja normalmente expressa em $\mathrm{mL}(\mathrm{CNTP}) \cdot \mathrm{m}^{-2} \cdot \mathrm{dia}^{-1}$, no sistema internacional de unidades é expressa em mol. $\mathrm{m}^{-2} \cdot \mathrm{s}^{-1}$, onde $1 \mathrm{~mL}(\mathrm{CNTP})$ equivale a $44,62 \mu \mathrm{mol}$ e 1 dia são $86,4 \times 10^{3} \mathrm{~s}$.

\section{Permeabilidade e modelo de Nielsen}

Os valores médios de taxa de permeabilidade ao oxigênio $\left(\mathrm{TPO}_{2}\right)$ e ao vapor d'água (TPVA) estão apresentados na Tabela 4. Para eliminar os efeitos de diferenças de espessura das amostras, os valores de taxa de permeabilidade ( $\mathrm{TPO}_{2}$ e TPVA) foram convertidos para coeficiente de permeabilidade ( $\mathrm{PO}_{2}$ e PVA, respectivamente) e são apresentados também na Tabela 4 , juntamente com a redução observada para estes valores.

Para melhor identificação da importância relativa das variáveis estudadas na redução da permeabilidade foram feitos diagramas de Pareto referentes aos coeficientes de permeabilidade (Figura 3). Observou-se influência das variáveis estudadas na permeabilidade ao oxigênio e ao vapor d'água. $\mathrm{O}$ aumento no teor de argila diminuiu de forma significativa a permeabilidade ao oxigênio e ao vapor d'água, denotando o efeito do aumento de tortuosidade esperado no sistema. Já o teor de acetato promoveu aumento na permeabilidade, sendo mais pronunciado para o vapor d'água, pois a polaridade tem efeito na solubilidade da água (polar) no EVA, mas tem pouco efeito na solubilidade do oxigênio no EVA. O efeito do Acetato de Vinila na $\mathrm{PO}_{2}$ provavelmente advém do espaçamento entre as macromoléculas, que aumenta a difusibilidade do $\mathrm{O}_{2}$, o que já é visto quando se compara o $\mathrm{PO}_{2}$ do EVA 19 


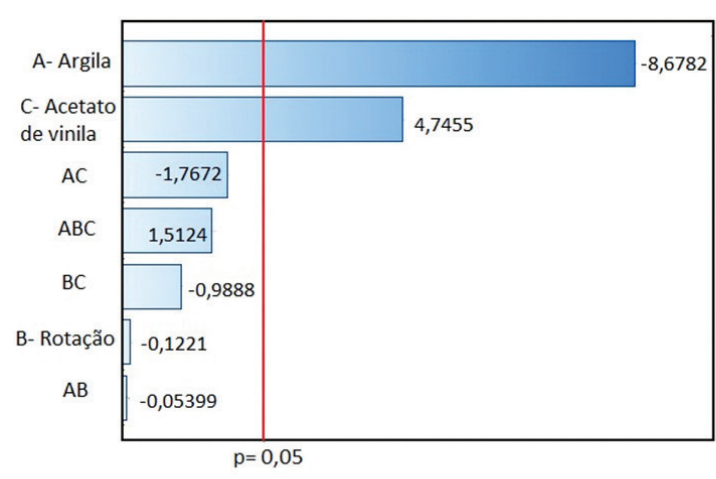

(a)

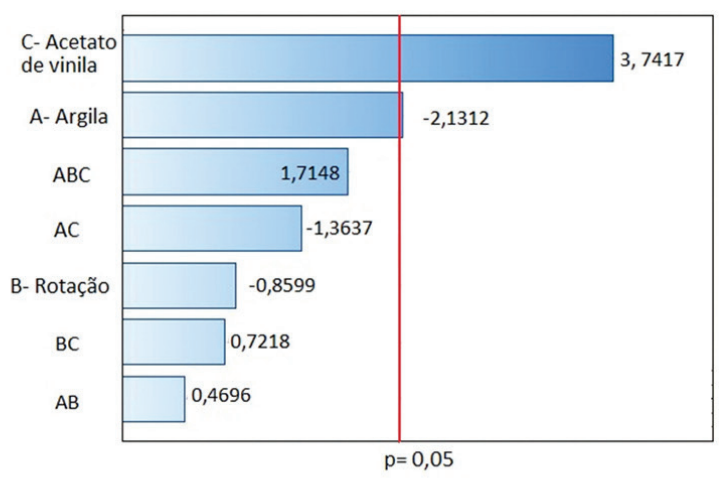

(b)

Figura 3. Diagramas de Pareto. (a) $\mathrm{PO}_{2}$; (b) PVA.

Tabela 5. Valores de razão de aspecto do EVA19, EVA28 e seus nanocompósitos.

\begin{tabular}{lcc}
\hline \multicolumn{1}{c}{ Amostras } & $\alpha\left(\mathbf{P O}_{2}\right)$ & $\alpha(\mathbf{P V A})$ \\
\hline EVA19 & - & - \\
$\operatorname{EVA}(2 / 60 / 19)$ & 104 & 205 \\
$\operatorname{EVA}(5 / 60 / 19)$ & 97 & 67 \\
$\operatorname{EVA}(2 / 100 / 19)$ & 83 & 312 \\
$\operatorname{EVA}(5 / 100 / 19)$ & 103 & 174 \\
$\operatorname{EVA28}$ & - & - \\
$\operatorname{EVA}(2 / 60 / 28)$ & 27 & 55 \\
$\operatorname{EVA}(5 / 60 / 28)$ & 96 & 190 \\
$\operatorname{EVA}(2 / 100 / 28)$ & 59 & 244 \\
EVA(5/100/28) & 92 & 122 \\
\hline
\end{tabular}

EVA19: 19\% de Acetato de Vinila; EVA28: 28\% o de Acetato de Vinila; EVA (teor de argila/ rotação/ concentração de Acetato de Vinila.

com o do EVA 28. A variação da rotação, por sua vez, não implicou em respostas estatisticamente significativas sobre as propriedades de barreira.

Os valores de coeficiente de permeabilidade dos nanocompósitos foram calculados e utilizados para a aplicação do modelo de Nielsen, permitindo a determinação da razão de aspecto $\alpha$ (Tabela 5). Para isto, primeiramente foi necessário determinar para cada composição a fração volumétrica $\varphi$, calculada com base na fração inorgânica de argila e na massa específica da argila não modificada $\left(2,86 \mathrm{~g} / \mathrm{cm}^{3}\right)^{[24]}$. Para ambos os parâmetros, coeficiente de permeabilidade e razão de aspecto, nenhum dos fatores avaliados foi estatisticamente significativo.

O cálculo da razão de aspecto na forma proposta é uma forma de medir indiretamente o estado de esfoliação da argila, pois a permeabilidade irá variar em função das camadas impermeáveis que aumentam a tortuosidade do sistema dificultando a difusão dos gases. Quando as camadas da argila estão dispersas e melhor distribuídas reduzem a permeabilidade e pela Equação 1, maior será a razão de aspecto obtida. Já na forma de tactóides, menor será a quantidade de camadas impermeáveis distribuídas e a razão de aspecto assim calculada, será menor. A modificação da Equação 1 foi proposta por Nazarenko et al. ${ }^{[25]}$ onde avaliaram diferentes condições de dispersão de argila em poliestireno e apresentaram que a eficiência na redução da permeabilidade é função do número de camadas de argila nos tactóides. Isto é, para a argila totalmente esfoliada a eficiência é máxima, pois $\mathrm{W}$ é a espessura real de uma nanocamada de argila, mas quando há a presença dos tactóides W passa a representar a espessura do tactóide que pode conter várias nanocamadas agregadas.

No estudo conduzido por Ploehn e $\mathrm{Liu}^{[26]}$, que analisaram a esfoliação de montmorilonita por microscopia de força atômica, foram verificados valores de razão de aspecto variando entre 60 e 500 com valores médios de 166 e desvio de 86, e espessura da nanocamada de $1 \mathrm{~nm}$. Os valores obtidos de $\alpha$ mostram que existem variações na condição de dispersão e distribuição da argila, indicando que houve esfoliação parcial da argila na matriz de EVA.

\section{Conclusões}

Nanocompósitos de EVA e argila organofílica preparados pela técnica de intercalação do fundido mostraram uma estrutura intercalada (Difração de raios-X) e parcialmente esfoliada (modelo de Nielsen). A reometria de torque mostrou-se eficaz para preparação dos nanocompósitos, para a qual o aumento da rotação implicou em diminuição da resistência a tração e aumento da deformação na ruptura, e a variação da rotação não implicou em diferenças estatisticamente significativas sobre os valores de permeabilidade avaliados. A variação da polaridade do EVA, conseqüência do aumento do teor de Acetato de Vinila de 19\% para 28\%, não influenciou no espaçamento basal da argila dos nanocompósitos, o que pode estar associado à similaridade da viscosidade dos polímeros empregados, porém alterou as propriedades de barreira aumentando a permeabilidade ao oxigênio e ao vapor d'água dos materiais. Além disto, o aumento da polaridade reduziu o tempo de fusão durante o processamento e aumentou a flexibilidade do sistema, pois, diminuiu o módulo elástico e aumentou a deformação de ruptura. $\mathrm{O}$ teor de argila organofílica ( $2 \%$ e $5 \%)$ não modificou a estabilidade térmica dos nanocompósitos, e a estrutura resultante não foi alterada, pois foram observados valores muito próximos para o espaçamento basal da argila. $\mathrm{O}$ aumento da concentração da argila aumentou o módulo elástico, reduziu a deformação de ruptura e reduziu significativamente o coeficiente de permeabilidade ao oxigênio e ao vapor d'água. 


\section{Agradecimentos}

Os autores agradecem ao Laboratório de DRX do IPENUSP, pela análise de Difração de raios-X e ao CNPq pelas bolsas de Mestrado e Iniciação Científica.

\section{Referências Bibliográficas}

1. Melo, T.J.A.; Neves, G.A.; Araújo, E.M.; Araújo, W.D.; Brasileiro, M.I.; Rodrigues, A.W., A. W. - Polímeros, 17, p.219 (2007). http://dx.doi.org/10.1590/S0104-14282007000300011

2. Guimarães, T.R.; Morales, A.R.; Paiva, L. B. - Polímeros, 16, p.136 (2006). http://dx.doi.org/10.1590/S010414282006000200014

3. Gorrassi, G.; Tortora, M.; Vittoria ,V.; Kaempferb, D. \& Mülhaupt, R. - Polymer (Guildf.)., 44, p.3679 (2003). http:// dx.doi.org/10.1016/S0032-3861(03)00284-2.

4. Krishnamachari, P.; Zhang, J.; Lou, J.; Yan, J. \& Uitenham, L. - Int. J. Polym. Anal. Charact., 14, p.336 (2009). http:// dx.doi.org/10.1080/10236660902871843.

5. Okamoto, M. - "Biodegradable polymer/layered silicate nanocomposites: a review”, in: Handbook of Biodegradable Polymeric Materials and Their Applications, cap. 8, Surya Mallapragada and Balaji Narasimhan (eds.), American Scientific Publishers, Valencia (2005).

6. Peacock, A. J. - "Handbook of polyethylene: structures, properties and applications", Marcel Dekker Inc., New York (2000).

7. Gianelli, W.; Camino, G., Dintcheva, N. T.; Verso, S. L. \& La Mantia, F. P. - Macromol. Mater. Eng., 289, p.238 (2004). http://dx.doi.org/10.1002/mame.200300267.

8. LaMantia,F.P.\&Dintcheva, N. T.-Polym. Test., 25,p.701 (2006). http://dx.doi.org/10.1016/j.polymertesting.2006.03.003.

9. Nawani, P.; Burger, C.; Rong, L.; Chu, B.; Hsiao, B. S.; Tsou, A. H. \& Weng, W. - Polymer., 51, p.5255 (2010). http://dx.doi. org/10.1016/j.polymer.2010.08.048.

10. Beltrán, M. I.; Benavente, V.; Marchante, V. \& Marcilla, A. - Appl. Clay Sci., 83-84, p.153 (2013). http://dx.doi. org/10.1016/j.clay.2013.08.028.

11. Morales, A. R.; Cruz, C. V. M.; Peres, L. \& Ito, E. N. Polímeros, 20, p.39 (2010). http://dx.doi.org/10.1590/S010414282010005000004 .
12. Nielsen, L. E. - J. Macromol. Sci. Chem. A1, 5, p.929 (1967).

13. Utracki, A. - "Clay-Containing Polymeric Nanocomposites", Rapra Technology, Shrewsbury (2004).

14. Choudalakis, G. \& Gotsis, A. D. - Eur. Polym. J., 45, p.967 (2009). http://dx.doi.org/10.1016/j.eurpolymj.2009.01.027.

15. Santos, P. S. - "Ciência e Tecnologia de Argilas", Ed. Edgard Blucher, São Paulo (1989).

16. Mandalia, T. \& Bergaya, F.-J. - Phys. Chem. Solids., 67, p. 836 (2006). http://dx.doi.org/10.1016/j.jpcs.2005.12.007.

17. Shafiee, M. \& Ramazani, A. - Macromol. Symp., 274, p.1 (2008). http://dx.doi.org/10.1002/masy.200851401.

18. Marini, J.; Branciforti, M. C. \& Lotti, C. - Polym. Adv. Technol., 21, p.408 (2010).

19. Zanetti, M. - Polymer., 42, p.4501 (2001). http://dx.doi. org/10.1016/S0032-3861(00)00775-8.

20. Ardhyananta, H.; Ismail, H. \& Takeichi, T. - J. Reinf. Plast. Compos., 26, p.789 (2007). http://dx.doi. org/10.1177/0731684407076722.

21. Chaudhray, D. S.; Prasad, R.; Gupta, R. K. \& Bhattacharya, S. N. - Thermochim. Acta., 433, p.187 (2005a). http://dx.doi. org/10.1016/j.tca.2005.02.031.

22. Chaudhary, D. S.; Prasad, R.; Gupta, R. \& Bhattacharya, S. N. - Polym. Eng. Sci., 45, p.889 (2005b). http://dx.doi. org/10.1002/pen.20349.

23. La Mantia, F. P.; Verso, S. L. \& Dintcheva, N. T. - Macromol. Mater. Eng., 287, p.909 (2002). http://dx.doi.org/10.1002/ mame.200290019.

24. Alexandre, B.; Langevin, D.; Médéric, P.; Aubry, T.; Couderc, H.; Nguyen, Q. T.; Saiter, A. \& Marais, S.-J. Membr. Sci., 328, p.186 (2009). http://dx.doi.org/10.1016/j. memsci.2008.12.004.

25. Nazarenko, S.; Meneghetti, P.; Julmon, P.; Olson, B. G. \& Qutubuddin, S. - Polym. Sci. Pol. Phys., 45, p.1733 (2007). http://dx.doi.org/10.1002/polb.21181.

26. Ploehn, H. J. \& Liu, C. - Ind. Eng. Chem. Res., 45, p.7025 (2006). http://dx.doi.org/10.1021/ie051392r.

Enviado: Out. 21, 2013

Reenviado: Fev. 17, 2014

Aceito: Abr. 16, 2014 